\title{
Mining Relational Association Rules for Propositional Classification
}

\author{
Annalisa Appice, Michelangelo Ceci, and Donato Malerba \\ Dipartimento di Informatica, Università degli Studi, \\ via Orabona, 4, 70126 Bari, Italy \\ \{appice, ceci, malerba\}@di.uniba.it
}

\begin{abstract}
In traditional classification setting, training data are represented as a single table, where each row corresponds to an example and each column to a predictor variable or the target variable. However, this propositional (featurebased) representation is quite restrictive when data are organized into several tables of a database. In principle, relational data can be transformed into propositional one by constructing propositional features and performing classification according to some robust and well-known propositional classification methods. Since propositional features should capture relational properties of examples, multi-relational association rules can be adopted in feature construction. Propositionalisation based on relational association rules discovery is implemented in a relational classification framework, named MSRC, tightly integrated with a relational database. It performs the classification at different granularity levels and takes advantage from domain specific knowledge in form of hierarchies and rules. In addition, a feature reduction algorithm is integrated to remove redundant features. An application in classification of real-world geo-referenced census data analysis is reported.
\end{abstract}

\section{Introduction}

Classification is one of the fundamental tasks in data mining and in the traditional data mining classification setting [16] data are generated independently and with an identical unknown distribution $P$ on some domain $\mathbf{X}$ and are labeled according to an unknown function $g$. The domain of $g$ is spanned by $m$ independent (or predictor) random variables $X_{i}$ (both numerical and categorical), that is $\mathrm{X}=\mathrm{X}_{1} \times \mathrm{X}_{2} \times \ldots \times \mathrm{X}_{\mathrm{m}}$, while the range of $g$ is a finite set $Y=\left\{\mathrm{C}_{1}, \mathrm{C}_{2}, \ldots, \mathrm{C}_{\mathrm{L}}\right\}$, where each $\mathrm{C}_{\mathrm{i}}$ is a distinct class. A data mining algorithm takes a training sample $S=\{(\mathbf{x}, \mathrm{y}) \in \mathbf{X} \times \mathrm{Y} \mid \mathrm{y}=\mathrm{g}(\mathbf{x})\}$ as input and returns a function $f$ which is hopefully close to $g$ on the domain $\mathbf{X}$. In practice, the goal is to learn the target concept associated with each class by finding regularities in examples of a class that characterize the class in question and discriminate it from the other classes. This formalization of the classification problem well faces with the single-table assumption [18] underlying most of classification methods proposed in the literature. Data are represented as a single table, where each row corresponds to an example and each column to a predictor variable or to the target variable $Y$. However, the growing importance of knowledge discovery and data mining in practical real world requires increasingly sophisticated solutions for classification problems when 
data consists of a large amount of records that may be stored in several tables of a relational database. In this context, single table assumption appears too restrictive since both the predictor variables and the target variable are represented as attributes of distinct tables (relations).

(Multi-) relational data mining [18] is the branch of data mining research that overcomes limitations imposed by single table assumption and investigates methods able to extract knowledge from data stored in multiple relational tables. (Multi-) relational data mining methods are typically based on two alternative approaches: a structural approach and a propositional approach. While in the first case the whole hypothesis space is directly explored by the mining method, in the second case, known as propositionalisation, a transformation of relational learning problems into attribute-value representations amenable for conventional data mining methods is performed. In principle, methods implementing structural approaches are more powerful than methods implementing propositional approaches since information about how data were originally structured is not lost. Nevertheless, approaches to (multi-)relational data mining based on propositionalisation have gained significant interest in the last few years. This is mainly due to the fact that in many practical cases propositionalisation allows the reduction of the search space to a minimal subset including features obtained as transformation of the original (multi-)relational feature space. Another reason is given by the observation that the transformation of an original (multi-)relational problem into a single table format allows one to directly apply conventional propositional data mining methods, thus making a wider choice of robust and well-known algorithms available [10].

Several multi-relational data mining methods based on propositionalisation have been proposed in literature for classification tasks. Generally, they assume an individual-centered data transformation such that there is a one-to-one correspondence between each tuple in the original target table (table containing the target attribute) and each tuple in the single table obtained after the propositionalisation process. At now, multi-relational classification through propositionalization problems has been extensively investigated by resorting to the field of Inductive Logic Programming (ILP). For instance, LINUS [11] as well as its successor SINUS [10] and ST [9] are able to construct a set of boolean features defined in terms of conjunctions of literals starting from relational data expressed in some first-order formalism. Nevertheless, a different approach to propositionalisation is supported by systems that directly work with relational databases. Indeed, they generally construct a single central relation by simply summarizing and/or aggregating information found in other tables and performing join operations according to foreign key constraints [8]. In both cases, propositional features are constructed by considering the structure imposed on data by relationships (e.g. foreign key constrains) between tuples in the target table and tuples in related tables, while ignoring the structure eventually imposed by hierarchical relationships on tuples of the same table. This means that these methods are not able to derive a propositional description of the same individual exploiting different levels of granularity. This turns to be an interesting aspect in many applications that would benefit from concept hierarchies [7]. Another limitation in the existing approaches is the huge number of new features they produce. In particular, propositionalisation tends to produce large numbers of features, many of which are highly correlated or even logically redundant. 
A simple example of a redundant feature is one that is never (or always) satisfied: e.g., 'a molecule having an atom which has a bond with itself'. While some forms of redundancy can be recognised at feature generation time, others can only come to light by examining the data [2].

In this paper we propose a multi-relational propositionalisation-based classification framework that makes use of discovered multi-level association rules in the propositionalisation step. Similarly to [3], association rules are extracted by means of a (multi-) relational association rule discovery system embedded in the framework that is able to generate association rules at multiple levels of granularity according to some domain knowledge. This allows the classification at different levels of granularity by taking advantage from a qualitative reasoning on a domain specific knowledge expressed in form of rules. Discovered rules are subsequently used to create a relational table for each granularity level where each column represent a boolean feature. Moreover, a feature reduction algorithm has been integrated to remove redundant features and improve efficiency of classification step without affecting accuracy of classifier. Finally, four well-known data mining algorithms are applied to the resulting table for classification purposes.

The paper is organized as follows. In the next section we present the architecture of the proposed framework. In section 3 we briefly present the process of mining of multi-level relational association rules while the propositionalization approach is described in Section 4. Finally, an application to in real-world geo-referenced census data analysis is reported in Section 5 and some conclusions are drawn.

\section{System Architecture}

The framework we present in this paper has been implemented in the system MSRC (Multi-Step Relational Classifier).

The problem solved by MSRC can be formalized as follows: Given: A training set represented by means of $h$ relational tables $S=\left\{T_{0}, T_{1}, \ldots, T_{h-1}\right\}$ of a relational database $D$; A set of primary and foreign key constraints on tables in $S$; a target relation (table) $T \in S$; A target discrete attribute $y$ in $T$, different from the primary key of $T$; A background knowledge $B K$ including some hierarchies $H_{k}, k \in\{j \in \mathcal{N} \mid 0 \leq j \leq h-1 \wedge$ $\left.T_{j} \neq T\right\}$ (one for each table $T_{k} \in S$ - $\{T\}$ ) on tuples in $S$ - $\{T\}$. Hierarchies $H_{k}$ define $i s$ - $a$ (i.e., taxonomical) relationship of objects represented as tuples in the table $T_{k} ; M$ granularity levels in the descriptions ( 1 is the highest while $M$ is the lowest); A set of granularity assignments $\psi_{k}$ which associate each object in $H_{k}$ with a granularity level; a couple of thresholds minsup $[l]$ and minconf $[l]$ for each granularity level. Find multilevel classifiers that predict $y$ for some individual represented as a tuple in $T$ (with possibly UNKNOWN value for y) and related tuples in $S$ according to foreign key constraints.

In Figure 1, the architecture of the multi-relational propositionalization-based classifier MSRC is presented. The MRSC Engine manages the system by allowing user interaction and by coordinating the activity of all other components. It interfaces the system with the database module in order to store intermediate information.

The Association rules discovery module is in charge of the extraction of association rules from data. In particular, given a database schema, a background knowledge and 
a Language Bias, the system is able to extract association rules and store them in an appropriate repository for subsequent use. The system embeds, for this task, SPADA [1] that mines association rules at multiple levels of granularity. The expressive power of the language bias of SPADA allows us to filter out rules that are not useful for classification purposes (see section 3).

The Propositionalization Module is in charge of the transformation of the tables in $S$ in a single relational table. In the resulting table, columns represent the extracted rules, rows represent instances of the target table $T$ and values are obtained by means of the propositionalization step (see section 4).

The Feature Reduction Module reduces the number of columns in order to remove redundant features that would uselessly increase the complexity of the learning task and negatively affect the classification effectiveness (see section 4).

Finally, the Classification Module is in charge of executing both the learning process and the classification process. The learning process takes as input the reduced relational table and returns a classification model. The classification process, takes in input the classification model and a tuple in $T$ (with possibly UNKNOWN value for y) and returns the value of $y$. The classification model can be computed by means of four different learning algorithms, namely the JRIP[4], C4.5 [17], 1-NN and the Naive Bayesian classifier (NBC) [5].

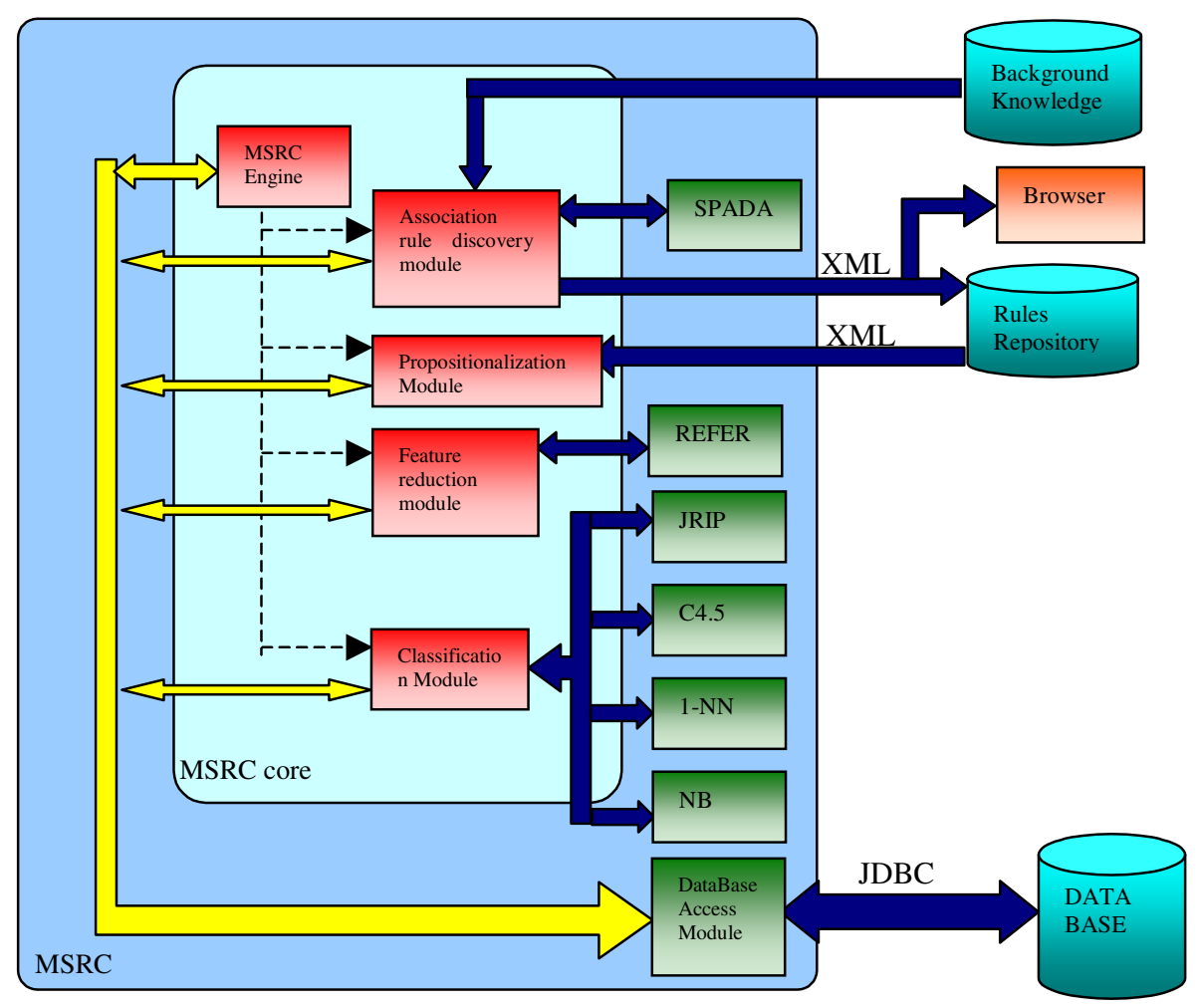

Fig. 1. MSRC architecture 
In the following Sections, we first introduce the problem of discovering multi-level relational association rules and then we describe how these rules can be used to construct boolean features to be used in the classification step.

\section{Relational Association Rules Mining}

The discovery of relational association rules is a descriptive mining task that aims at detecting associations between target objects (represented as tuples of the target table $T$ ) and some target-relevant objects (represented as tuples in $S$ - $\{T\}$ ). The former are the main subject of the description, while the latter are objects that are relevant for the task in hand and are related (by means of foreign key constraints) to the former.

In general, association rules are a class of regularities that can be expressed by the implication: $P \Rightarrow Q(s, c)$, where $P$ (body) and $Q$ (head) are a set of literals, called items, such that $P \cap Q=\varnothing$, the support $s$ estimates the probability $p(P \cup Q)$, and the confidence $c$, estimates the probability $\mathrm{p}(Q \mid P)$. The conjunction $\mathrm{P} \wedge \mathrm{Q}$ is called pattern. An example of relational association rule is:

is_a(X, molecule $)$, contains $(X, Y), i s \_a(Y$, atom $)$, charge_of $(Y,[0.7 \ldots 0.88]) \Rightarrow$ contains $(X, Z)$, is_a(Z, atom), charge_of(Z, $[0.2 \ldots 0.3]), Z \neq Y(91 \%, 100 \%)$

to be read as "If a molecule $X$ contains an atom with charge between 0.7 and 0.88 , then $\mathrm{X}$ contains another atom $\mathrm{Z}$ distinct from $\mathrm{Y}$ with charge between 0.2 and 0.3 with $91 \%$ support and $100 \%$ confidence." By taking into account some kind of taxonomic knowledge on target-relevant objects (e.g. atom is specialized as atom_A and atom_B) it is possible to obtain descriptions at different granularity levels (multiplelevel association rules). For instance, a finer-grained association rules can be:

is_a(X, molecule), contains $(X, Y)$, is_a( $Y$, atom_A $),$ charge_of $(Y,[0.7 \ldots 0.88])$

$\Rightarrow$ contains $(X, Z)$, is_a(Z, atom_B $)$, charge_of $(Z,[0.2 \ldots 0.3]), Z \neq Y(65 \%, 85 \%)$

In this context, the process of discovery multi-level relational association rules correspondes to mine strong multi-level relational association rules, that is, relational association rules involving target-relevant objects at different granularity levels.

Hierarchies $H_{k}$ define is- $a$ (i.e., taxonomical) relationship of objects in the same table (e.g. atom-A $i s$ - $a$ atom). Objects of each hierarchy are mapped to one or more of the $M$ user-defined description granularity levels in order to deal uniformly with several hierarchies at once. Both frequency of patterns and strength of rules depend on the granularity level $l$ at which patterns/rules describe data. Therefore, a pattern $P(s \%)$ at level $l$ is frequent if $s \geq \operatorname{minsup}[l]$ and all ancestors of $P$ with respect to $H_{k}$ are frequent at their corresponding levels. An association rule $Q \rightarrow R(s \%, c \%)$ at level $l$ is strong if the pattern $Q \cup R(s \%)$ is frequent and $\mathrm{c} \geq \operatorname{minconf}[l]$.

In MSRC, rules are extracted by means of the algorithm SPADA [13] that operates in three steps for each granularity level: i) pattern generation; ii) pattern evaluation; iii) rule generation and evaluation. SPADA takes advantage of statistics computed at granularity level $l$ when computing the supports of patterns at granularity level $l+1$. In particular, SPADA exploits the expressive power of first-order logic to specify both the background knowledge BK, such as hierarchies and domain specific knowledge, and the language bias LB. Hierarchies allow to represent and manage objects at 
different levels of granularity, while the domain specific knowledge stored as a set of rules supports qualitative reasoning. On the other hand, the $L B$ is relevant to allow data miners to specify his/her bias for interesting solutions, and then to exploit this bias to improve both the efficiency of the mining process and the quality of the discovered rules. In SPADA, the language bias is expressed as a set of constraint specifications for either patterns or association rules. Pattern constraints allow specifying a literal or a set of literals that should occur one or more times in discovered patterns. During the rule generation phase, patterns that do not satisfy a pattern constraint are filtered out. Similarly, rule constraints are used do specify literals that should occur in the head or body of discovered rules. In a more recent release of SPADA (3.1) [1] new pattern (rule) constraints have been introduced in order to specify exactly both the minimum and maximum number of occurrences for a literal in a pattern (head or body of a rule). An additional rule constraint has been introduced to eventually specify the maximum number of literals to be included in the head of a rule. In this way we are able to constraint the head structure of a rule requiring the presence of only the literal representing the class label and obtain patterns useful for classification purposes.

\section{Transforming Association Rules in Boolean Features}

Once relational association rules having only the class label in the head have been extracted for each level, they are converted in a set of boolean features such that the result can be used as input for attribute-value classification algorithms. More precisely, for each granularity level $l$, boolean features construction is performed by transforming the original set of tables $S$ in a single relational table $B$ whose columns correspond to each body of association rules discovered at level $l$. Before describing how $B$ is obtained, we introduce: $i) R^{\prime}$ : a set of relational association rules whose head contains a literal representing the class label; ii) $I_{i} \in T$ : a target object instance (tuple of $T$ ); iii) $B$ : the output table with $\left|R^{\prime}\right|+1$ attributes. Tuples in $B$ correspond to tuples in $T$.

The instance $I_{i}$ can be logically represented as a set of ground facts describing both the target tuple in $T$ and all target-relevant tuples that are related (e.g. foreign key associated) to the target table (see Figure 2). The body of a rule $R_{j} \in R^{\prime}$ covers $I_{i} \in T$ if there exists a substitution $\theta$, such that $\operatorname{body}\left(R_{j}\right) \theta \subseteq I$.

In the case that the body of the rule $R_{j}$ covers $I_{i}$, the $j$-th value of the tuple in $B$ associated to the $i$-th instance of $\mathrm{T}$ is true, otherwise it is false. The $\left(\left|R^{\prime}\right|+1\right)$-th column in $B$ represents the target attribute $y$.

It is noteworthy that the number of attributes of $B$ depends on the number of discovered association rules in $R$ '. Since, the number of discovered association rules is usually high and many rules are strongly correlated, this may lead to generate boolean features which are highly correlated or even logically redundant.

The definition of feature redundancy we adopt in this work properly follows from reducts in rough sets theory [15], in which boolean features are redundant if their removal does not change the set of example-pairs having the same value for each feature. Hence, a feature $f$ can be identified as redundant with respect to another feature $g$ for distinguishing positive from negative examples of a class $c$ if $f$ is true for at least the same positive examples as $g$, and false for at least the same negative 
examples as $g$. Coherently with this definition, we may combine the association rule based propositionalisation with redundant feature elimination (feature reduction) in order to reduce the hypothesis space by excluding boolean features (or literals in ILP) which are redundant for learning. For this purpose, we integrate REFER [2], that is, a feature reduction method for multi-class problems that operates in an ILP setting.

Central to REFER is the notion of a neighborhood that is a subset of examples belonging to the same class such that the number of different features between examples is relatively small. Each neighbourhood is uniquely identified by two examples. The first example is where the neighbourhood construction starts and the second one is the termination point. Let $e_{s}$ be a random starting example for the construction of a neighbourhood, the corresponding termination point is the closest example in $e_{t}$ tagged with a different class label, referred to here as the point (or example) of class change. The neighbourhood $E\left(e_{s}, e_{t}\right)$ contains the set of training examples $e_{s_{1}}, e_{s_{2}}, \ldots, e_{s_{k}}$ such that:

$\operatorname{class}\left(e_{s}\right)=\operatorname{class}\left(e_{s_{1}}\right)=\ldots=\operatorname{class}\left(e_{s_{k}}\right), \operatorname{distance}\left(e_{s}, e_{s_{h}}\right) \leq \operatorname{distance}\left(e_{s}, e_{t}\right), \forall h=1, \ldots, k$

where the distance between two examples is computed as the Hamming distance, that is, the number of features whose values differ between the two examples. The neighbourhood construction proceeds in $E \backslash E\left(e_{s}, e_{t}\right)$ by considering the last example of class change as the current starting point and the process is repeated until the entire set of training examples is partitioned in neighbourhoods.

Redundant features are then eliminated by applying a revised version of REDUCE [12] method to each pair of neighbourhoods of different class. In particular, let $E_{l}$ and $E_{m}$ be a pair of neighbourhoods in $E$ of different classes $\left(C_{l} \neq C_{m}\right)$, the goal is to detect which features $f \in B$ describing examples in $E_{l} \cup E_{m}$ are non-redundant for discriminating between the classes $C_{l}$ and $C_{m}$. We thereby eliminate features estimated redundant according to properties and dependencies among the features. Formally, a feature $f \in F$ covers a feature $g \in F$ with respect to $E_{l} \cup E_{m}$ if $T(g) \subseteq T(f)$ and $F(g) \subseteq F(f)$, where $T(f)(T(g))$ is the set of all examples $e_{i} \in E_{l}$ such that $f(g)$ has the value true for $e_{i}$ and $F(f)(F(g))$ is the set of all example $e_{j} \in E_{m}$ such that $f(g)$ has the value false for $e_{j}$. The intuition is that a feature $f$ is better than another feature $g$ for distinguishing $C_{l}$ from $C_{m}$ if $f$ is true for at least the same $C_{l}$ examples as $g$, and false for at least the same $C_{m}$ examples as $g$. The implicit assumption is that class $C_{l}$ is the positive class we are trying to describe. This suggests the notion of useless features,
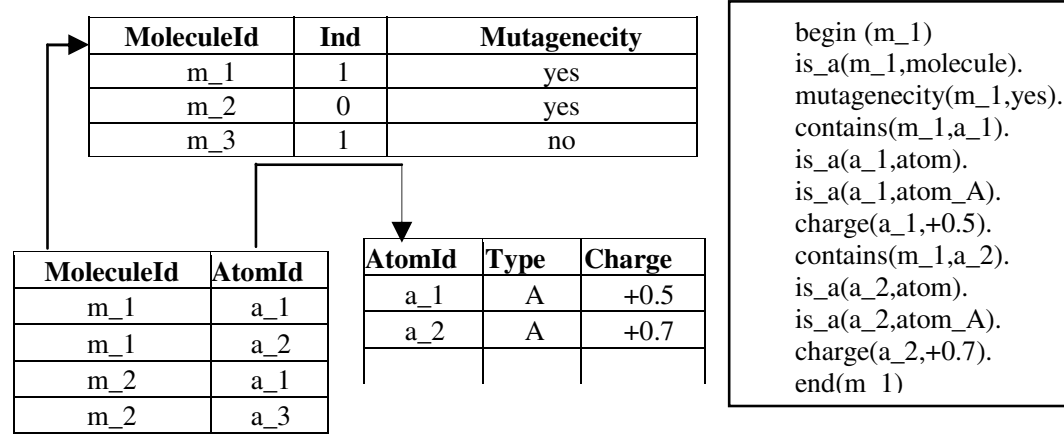

Fig. 2. Set of ground atoms representing relational data concerning molecules (target objects) and atoms (target-relevant objects). In this case a two level hierarchy is defined on atom, that is, atom is specialized as atom_A and atom_B. 
those for which $T(f)=\varnothing$ or $F(g)=\varnothing$. Such features can be immediately removed from the set of features $F$ regardless of the properties of other features. Furthermore, a feature $g \in F$ is considered as a redundant feature to be removed if there exists another feature $f \in F(f \neq g)$ such that $f$ covers $g$.

Since, it is proved that REFER preserves the existence of a complete and consistent theory for each class label when eliminating redundant features [2], this method appears well-suited to reduce the set of features describing data at each granularity level, thus improving efficiency of classification step without comprising the existence of some classification model discriminating between examples tagged with different class label.

\section{Experimental Results}

In this section we present a real-world application MSRC in geo-referenced census data interpretation. For this study we consider both census and digital map data provided in the context of the European project SPIN! (Spatial Mining for Data of Public Interest). These data concern Greater Manchester, one of the five counties of North West England (NWE). Greater Manchester is divided into ten metropolitan districts, each of which is decomposed into censual sections or wards, for a total of two hundreds and fourteen wards. Census data are available at ward level and provide socio-economic statistics (e.g. mortality rate, that is, the percentage of deaths with respect to the number of inhabitants) as well as some measures describing the deprivation level of each ward according to information provided by Census combined into single index scores. We consider Jarman Underprivileged Area Score that is designed to measure the need for primary care, the indices developed by Townsend and Carstairs that is used in health-related analyses, and the Department of the Environment's Index (DoE) that is used in targeting urban regeneration funds. The higher the index value the more deprived a ward is. Both deprivation indices values as well as mortality rate are all numeric, but discrete values are here obtained by applying the Relative Unsupervised DiscrEtization RUDE algorithm [14] that discretizes each continuous variable in the context defined by remaining continuous variables. More precisely, Jarman index, Townsend index, DoE index and Mortality rate have been automatically discretized in (low, high), while Carstairs index has been discretized in (low, medium, high).

By considering Greater Manchester wards as target objects, we focus our attention on mining a classification model to predict discrete value of DoE index by exploiting not only socio-economic factors represented in census data but also geographical factors represented in some linked topographic maps. Spatial analysis is then enabled by the availability of vectorized boundaries of the 1998 census wards as well as by other Ordnance Survey digital maps of NWE, where several interesting layers (road net, rail net, water net, urban area and green area) forming target-relevant objects are found (see Table 1). Both ward-referenced census data and map data are stored in an ObjectRelational spatial database, i.e., Oracle Spatial 9i database, as a set of spatial tables, one for each layer. Each spatial table includes a geometry attribute that allows storing the geometrical representation (e.g. point, line, and region in a $2 \mathrm{D}$ context) and the positioning of a spatial object with respect to some reference system in a single geometry field within a spatial table. 
Table 1. Geographic layers

\begin{tabular}{|c|c|c|c|}
\hline \multicolumn{2}{|c|}{ Layer name } & Geometry & Number of objects \\
\hline Road net & A-road; B-road; Motorway; Primary road & Line & 2798 \\
\hline Rail net & Railway & Line & 1054 \\
\hline Urban area & Large urban area; Small urban area & Line & 381 \\
\hline Green area & Wood; Park: & Line & 13 \\
\hline Water net & Water; River; Canal & Line & 1067 \\
\hline Ward & Ward & Region & 241 \\
\hline
\end{tabular}

It is noteworthy that the spatial nature of both target objects and target-relevant objects poses two main degree of complexity that is the granularity of the spatial objects and the implicit definition of spatial relations. The former is due to the fact that non-target objects can be described at multiple levels of granularity. In this case, five different hierarchies can be defined to describe target-relevant layers (see Fig. 3). The hierarchies have depth three and are straightforwardly mapped into three granularity levels. The second source of complexity refers to the fact that both geometrical representation and relative positioning define implicitly spatial relations of different nature (e.g. topological) which can be explicitly modeled as many relational tables as the number of objects type and spatial relations. Modeling these spatial relations has a key role in this classification problem, since both the attribute values of the object to be classified and the attribute values of spatially related objects may be relevant for assigning an object to a class from the given set of classes.

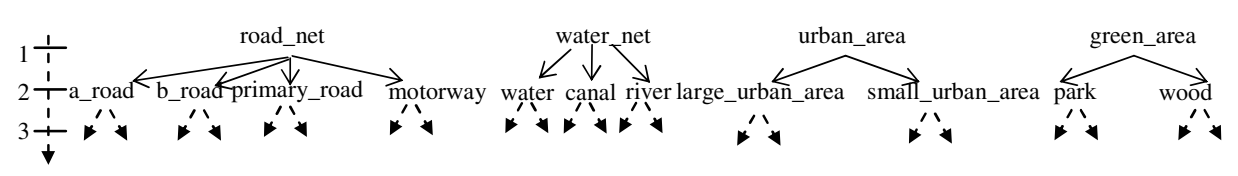

Fig. 3. Spatial hierarchies defined for road net, water net, urban area and green area

We adopt here some geometrical and topological algorithm based on the 9intersection model [6] to extract topological relationships between target objects and target-relevant objects. For instance the relationship crosses(ward_135, urbareaL_151) denotes that ward_135, that is a specific Greater Manchester ward, is crossed by urbanareaL_151 that is a large urban area. The number of computed relationships is 784,107 . To support a spatial qualitative reasoning, a domain specific knowledge (BK) is expressed in form of a set of rules. Some of these rules are:

ward_urbanarea $(X, Y):-i s \_a(X, w a r d)$, connects $(X, Y)$, is_a(Y, urban_area $)$. ... ward_urbanarea $(X, Y)$ :- is_a(X,ward), inside $(X, Y)$, is_a(Y,urban_area).

The use of the predicate is_a hides the fact that a hierarchy is defined for spatial objects which belong to the urban area layer. According to spatial relations introduced with BK, we easily obtain a relational representation of classification problem in question (see Figure 4).

Performances of MSRC are evaluated by means of a 10 -fold cross validation. The first step consists in the extraction of association rules at different levels of granularity according to hierarchies defined on target-relevant objects. Rules contain useful 
information about relational patterns frequently occurring in data. For instance, by analyzing spatial association rules extracted with parameters minsup $=0.1$, minconf $=$ 0.6 we discover the following rule:

$$
\begin{gathered}
\text { doe }(A, \text { low }) \leftarrow \text { is_a }(A, \text { ward }), \text { ward_urbanarea }(A, B), \\
\text { is_a }(B, \text { urban_area }), \text { jarman }(A, \text { low }) \quad(52.6 \%, 100 \%)
\end{gathered}
$$

which states that a low DoE index value is observed in a ward $A$ that includes an urban area $B$ and has a low value of Jarman index. The support (52.6\%) and the high confidence $(100 \%)$ confirm a meaningful association between a geographical factor, such as living in urban areas where primary care are well satisfied, and low level of derivation when considering targeting urban regeneration funds. In the feature construction step, this rule defines the boolean feature "is_a(A, ward)^ ward_urbanarea $(A, B) \wedge i s \_a(B$, urban_area $) \wedge j \operatorname{jarman}(A$, low $)$ " that is true for each Greater Manchester ward with a low value of Jarman index such that there is at least an urban area that is topologically related (e.g. connects or inside) to the ward in question. At a granularity level 2, SPADA specializes the target-relevant object B by generating the following rule that preserves both support and confidence:

$$
\begin{gathered}
\text { doe }(A, \text { low }) \leftarrow i s \_a(A, \text { ward }), \text { ward_urbanarea }(A, B), \\
\text { is_a }(B, \text { large_urban_area }), \text { jarman }(A, \text { low })
\end{gathered}
$$

This rule clarifies that the urban area B is large. By varying granularity level as well as the value of minsup, minconf and the number of refinement steps $K$ (pattern length) in association rule discovery, we obtain several experimental settings. For each setting, the set of discovered rules is transformed in a set of boolean features that is the conjuctions of literals derived from the body of each rule, and redundant features are removed to reduce feature space without affecting the existence of a complete and consistent theory for each class label. Finally, we apply the four classification learners embedded in MSRC and compare the percentage of correct classification averaged over 10 -folds of cross-validation Results on average reduction percentage and average accuracy are reported in both Table 2 . Results confirm that high number of association rules typically lead to redundant features. Percentage of feature reduction increases when the refinement step increases. This is due to the high number of similar rules (thus

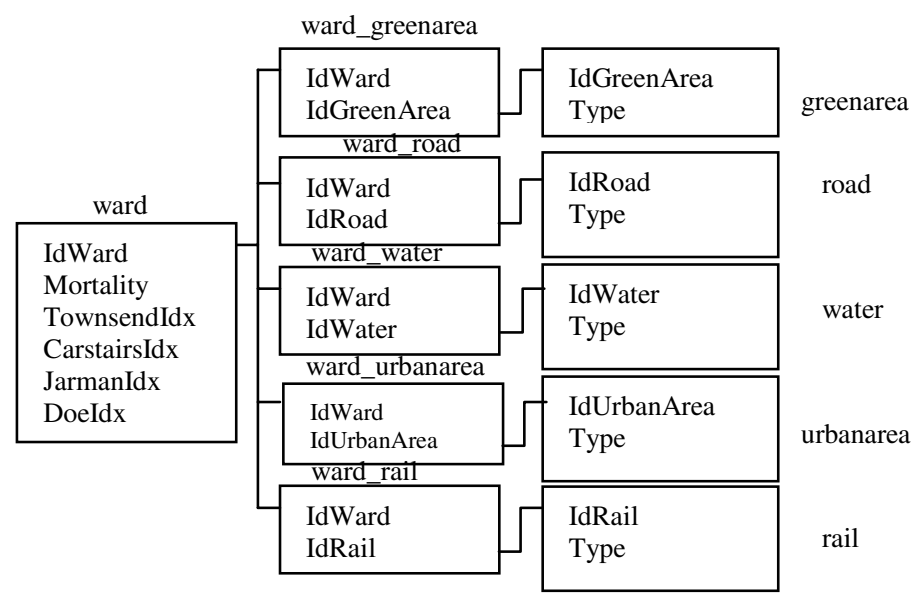

Fig. 4. Relational data describing ward-referenced census data and map data 
producing redundant features). Another consideration is that the average predictive accuracies of classification models discovered at higher granularity levels (i.e. level=2) are sometimes better than the corresponding accuracies at lowest levels. This means that the classification model takes advantage of the use of the hierarchies defined on spatial objects. In this case, results at different abstraction levels provide insights on what are the non-target objects that affect the classification. For instance, when $K=7$, minsup $=$ 0.1 and minconf $=0.6$, naïve-bayes classification is strongly improved when considering the size of the urban area, the type of road, and so on. Moreover, results show that by decreasing the number of extracted rules (higher support and confidence) we have lower accuracy. This means that there are boolean features that strongly influence classification results and often these features correspond to rules which are not characterized by high values of support and confidence. Finally, we observe that, in this specific task, the higher the number of refinement steps (boolean features involving more literals) not necessarily means the better the model. This is mainly due to the fact that rules become very specific.

Table 2. DoE Index average accuracy

\begin{tabular}{|c|c|c|c|c|c|c|c|c|c|}
\hline \multirow{2}{*}{$\begin{array}{l}\min \\
\text { sup }\end{array}$} & \multirow{2}{*}{$\begin{array}{l}\min \\
\operatorname{conf}\end{array}$} & \multirow{2}{*}{$\begin{array}{c}\text { Granularity } \\
\text { level }\end{array}$} & \multirow{2}{*}{$\mathrm{K}$} & \multirow{2}{*}{$\begin{array}{c}\text { Avg. number } \\
\text { of original } \\
\text { features }\end{array}$} & \multirow{2}{*}{$\begin{array}{l}\text { Avg. perc. of } \\
\text { feature } \\
\text { reduction }\end{array}$} & \multicolumn{4}{|c|}{ Avg. Accuracy } \\
\hline & & & & & & NBC & $1-\mathrm{NN}$ & $\mathrm{C} 4.5$ & JRIP \\
\hline 0.2 & 0.8 & \multirow{3}{*}{1} & 5 & 27.5 & $37 \%$ & 81.64 & 80.73 & \begin{tabular}{|l|}
83.01 \\
\end{tabular} & 83.01 \\
\hline 0.2 & 0.8 & & 6 & 221.1 & $44 \%$ & 80.28 & 80.73 & 83.01 & 83.01 \\
\hline 0.2 & 0.8 & & 7 & 982.3 & $73 \%$ & 75.56 & 80.28 & 83.01 & 83.01 \\
\hline 0.2 & 0.8 & \multirow{3}{*}{2} & 5 & 492.8 & $29 \%$ & 82.55 & 82.55 & 81.64 & 81.93 \\
\hline 0.2 & 0.8 & & 6 & 278.3 & $11 \%$ & 82.10 & 82.55 & 82.10 & 83.01 \\
\hline 0.2 & 0.8 & & 7 & 1860.1 & $66 \%$ & 80.28 & 82.55 & 81.19 & 81.02 \\
\hline 0.1 & 0.6 & \multirow{3}{*}{1} & 5 & 233.2 & $48 \%$ & 83.01 & 80.73 & 83.01 & 83.01 \\
\hline 0.1 & 0.6 & & 6 & 374 & $57 \%$ & 80.28 & 80.73 & 83.01 & 83.01 \\
\hline 0.1 & 0.6 & & 7 & 904.2 & $74 \%$ & 80.73 & 80.73 & 83.01 & 83.01 \\
\hline 0.1 & 0.6 & \multirow{3}{*}{2} & 5 & 442.2 & $28 \%$ & \begin{tabular}{|l|}
83.01 \\
\end{tabular} & 81.30 & 82.10 & 81.02 \\
\hline 0.1 & 0.6 & & 6 & 635.8 & $27 \%$ & 82.55 & 79.65 & 80.28 & 81.64 \\
\hline 0.1 & 0.6 & & 7 & 3051.4 & $68 \%$ & 80.73 & 79.31 & 81.19 & 81.02 \\
\hline
\end{tabular}

\section{Conclusions}

In this paper we have presented a novel relational classification framework named MSRC (Multi-Step Relational Classifier) that exploits relational multi-level association rules discovery to perform relational classifications at multiple level of granularity. Multi-level relational association rules are mined by taking advantage from a qualitative reasoning supported by domain specific knowledge in form of rules. For each level, relational rules are then transformed in boolean features making MSRC able to perform multi-level classification according to some robust and well-known propositional classification methods. Due to the high number of boolean redundant features constructed from association rules, MSRC integrates a feature reduction method that reduces feature space by removing redundant features without 
compromising classification effectiveness. The application in geo-referenced census data interpretation has confirmed that multi-level classification provides insights on what target-relevant objects really affect the classification and confirms benefits in considering concept hierarchies for classification purposes. Moreover, results show that support and confidence are not a valid criterion to filter rules, since rules characterized by low values of support and confidence positively affect classification results. As future work we intend to extend experimental evaluation by comparing MSRC with a (multi-)relational structural approach to multi-level classification.

\section{Acknowledgments}

We thank Simon Rawles and Peter Flach for working together in defining and developing the REFER algorithm. The work presented in this paper is partial fulfilment of the research objective set by the ATENEO-2005 project on "Gestione dell'informazione non strutturata: modelli, metodi e architetture".

\section{References}

[1] Appice, A., Berardi, M., Ceci, M., Lapi, M., Malerba, D., Turi, A: Mining interesting spatial association rules: two case studies. 86-97 (2004).

[2] Appice, A., Ceci, M. Rawles, S., Flach, P.: Redundant Feature Elimination for MultiClass Problems. Proc. of International Conference on Machine Learning, 33-40, (2004).

[3] Ceci, M., Appice, A., Malerba, D.: Spatial Associative Classification at Different Levels of Granularity: A Probabilistic Approach. Proc of European Conference on Principles and Practice of Knowledge Discovery in Databases, LNAI 3202, 99-111, (2004).

[4] Cohen, W. W.. Fast Effective Rule Induction. Proc. of the International Conference on Machine Learning, 115-123, Morgan Kaufmann (1995).

[5] Domingos, P. \& Pazzani, M.: On the optimality of the simple Bayesian classifier under zero-one loss, 29, 103-130. (1997).

[6] Egenhofer, M.J.: Reasoning about Binary Topological Relations. Proc. of the Symposium on Large Spatial Databases, 143-160, (1991).

[7] Han J., Fu Y.: Discovery of multiple-level association rules from large databases., In 21st International Conference on Very Large Data Bases, VLDB'95, 420-431, (1995).

[8] Knobbe A.J., Haas M. \& Siebes A: Propositionalisation and aggregates. In 5th European Conf. on Principles of Data Mining and Knowledge Discovery, Springer-Verlag, (2001).

[9] Kramer S., Pfahringer B., and Helma C.: Stochastic Propositionalization of NonDeterminate Background Knowledge, Proc. of the International Conference on Inductive Logic Programming, LNCS 1446, 80-94, (1998).

[10] Krogel, M., Rawles, S., Zelezny, F., Flach, P., Lavrac, N., Wrobel S.: Comparative evaluation of approaches to propositionalization. Proc. of the International. Conference. on Inductive Logic Programming,. Springer-Verlag, 197-214, (2003).

[11] Lavrac N. and Dzeroski S.: Inductive Logic Programming: Techniques and Applications. Ellis Horwood, (1994).

[12] Lavrač, N., Gamberger, D., Jovanoski V.. A study of relevance for learning in deductive databases. The Journal of Logic Programming, 16, 215-249. (1999)

[13] Lisi, F.A., Malerba, D.: Inducing Multi-Level Association Rules from Multiple Relations. Machine Learning, 55, 175-210, (2004). 
[14] Ludl, M.C., Widmer, G.: Relative Unsupervised Discretization for Association Rule Mining. PKDD’00, LNCS 1910, 148-158, (2000).

[15] Modrzejewski, M.: Feature selection using roughsets theory. Proceedings of the European Conference on Machine Learning. Springer-Verlag,213-226, (1993).

[16] Mitchell, T. Machine Learning. McGraw Hill (1997)

[17] Quinlan, J.: C4.5: Programs for machine learning. Morgan Kaufmann (1993)

[18] Wrobel, S. Inductive logic programming for knowledge discovery in databases. In: Džeroski, S., N. Lavrač(eds.): Relational Data Mining, Springer: Berlin, 74-101, (2001). 